\title{
Fungal infection in foot diabetic patients
}

Omid Raiesi $^{* 1}$, Hajar Shabandoust ${ }^{2}$, Parvin Dehghan ${ }^{3}$, Sina Shamsaei ${ }^{4}$, Ameneh Soleimani ${ }^{5}$

1. Department of Medical Parasitology and Mycology, School of Public Health, Tehran University of Medical Sciences, Tehran, Iran

2. Department of Parasitology and Mycology, School of Medicine, Kerman University of Medical Sciences, Kerman, Iran

3. Department of Mycology and Parasitology, School of Medicine, Isfahan University of Medical Sciences, Isfahan, Iran

4. Department of Medical Mycology and Parasitology, School of Medicine, Iran University of Medical Sciences, Tehran, Iran

5. Department of Medical Parasitology and Mycology, School of Public Health, Mazandaran University of Medical Sciences, Sari, Iran

*Corresponding author:Tel: +98 9189436166 Fax: +98 8433622946

Address: Department of Medical Parasitology and Mycology, School of Public Health, Tehran University of Medical Sciences, Tehran, Iran

E-mail: omid_raissi69@yahoo.com

Received; 2018/01/16 revised; 2018/03/28 accepted; 2018/08/16

\section{Abstract}

Diabetic patients are more susceptible to cutaneous fungal infections. Cutaneous lesions and Foot infections are a frequent complication of patients with diabetes mellitus, accounting for up to $20 \%$ of diabetes-related hospital admissions. Foot ulcers and other foot problems are a major cause of morbidity and mortality in people with Diabetes mellitus. The higher blood sugar levels cause increasing the cutaneous fungal infections in these patients. More than $75 \%$ of diabetes mellitus (DM) patients are at risk for diabetic ulcers. About $15 \%$ of foot ulcers in diabetic patients lead to amputations. Although every 30 seconds one leg is amputated in the world due to DM, $80 \%$ of these cases are preventable. Poor controlled had significantly higher fungal infection in diabetic foot ulcers and require careful attention and management. The findings of various studies indicate that the prevalence of fungal infections in patients with diabetic foot ulcers is increasing and there are many drug resistance issues reported in this area, therefore more attention is important in diabetic centers about this neglected issue.

Keywords: Diabetic foot ulcer, Fungal infections, Diabetes mellitus (DM)

\section{Introduction}

Diabetes mellitus (DM) is the most common endocrine metabolic disease (1). The prevalence of diabetes mellitus in Iran has been reported $7-17 \%$ and is increasing in most populations $(2,3)$. Among persons diagnosed as having diabetes mellitus, the prevalence of foot ulcers is $4 \%$ to $10 \%$, the annual population-based incidence is $1.0 \%$ to $4.1 \%$, and the lifetime incidence may be as high as $25 \%$ (4).

Type 2 diabetes (T2D) is more common than type 1 diabetes (T1D). The predisposing factors, high blood glucose, vascular insufficiency, neuropathy and, various immunological disturbances, facilitate conditions for colonization of

Copyright (C) 2018 Journal of Basic Research in Medical Science. This is an open access article distributed under the terms of the Creative Commons Attribution 4.0 International License (https://creativecommons.org/licenses/by-nc/4.0/) which permits copy and redistribute the material, in any medium or format, provided the original work is properly cited. 
Pathogenic fungi; including Candida, Dermatophytes, Malassezia ,Zygomycetes, Aspergillus and Fusarium species in DM patients (4-6). Nearly $56 \%$ of diabetic foot ulcers become infected and $20 \%$ of these patients with infected foot ulcers land up in some type of foot amputation. Infections in diabetic foot ulcer are mostly polymicrobial, and risk of development of diabetic foot syndrome is more with mycotic infections. However, little data are available on the prevalence of fungal foot infections in patients with diabetes. The fungi involved in diabetic foot ulcers are mainly Candida spp. Other species commonly isolated are Trichophyton sp. and Epidermophyton floccosum (E. floccosum) (7).

Invasive fungal infections are major medical complications in immunocompromised patients. Although aspergillosis and candidiasis are among the most common invasive mycoses in such patients, there is evidence that the incidence of infectious diseases caused by Zygomycetes has risen significantly over the past decade. Zygomycosis is a term used to describe a group of fungal infections caused by pathogenic molds belonging to the class Zygomycetes of the phylum Zygomycota. Zygomycosis is no longer an uncommon disease, due in part to the continued rise of diabetes and the increased use of immunosuppressive agents in the current era of advanced modern medicine. The most common species causing angio-invasive zygomycosis is Rhizopus arrhizus (oryzae), followed by Rhizopus microsporus var. rhizopodiformis and Rhizomucor pusillus. Approximately half of all zygomycosis cases are caused by Rhizopus spp. $R$. oryzae is the most frequent cause of rhino-cerebral forms of zygomycosis, whereas $R$. microsporus var. rhizopodiformis produces primarily cutaneous and gastrointestinal zygomycosis. Absidia (Lichtheimia) corymbifera is the primary species isolated from the earliest case reports of zygomycosis and ranks as the second most common organism causing zygomycosis. Diabetic patients with sustained hyperglycemia, particularly those with ketoacidosis, are more susceptible to zygomycosis. In fact, diabetes mellitus as a predisposing factor has been reported in $36-88 \%$ of all zygomycosis cases(8). Therefore, screening and early detection of fungal infections in high-risk individuals، are critical for prevention of grave complications like foot amputation. In some diabetic patients, developing cutaneous lesions, and nail infections have been documented (9). More than $75 \%$ of DM patients are at risk for diabetic ulcers. A diabetic foot ulcer is one of the most important complications in diabetic patients. About $15 \%$ of foot ulcers in diabetic patients lead to amputations. Although every 30 seconds one leg is amputated in the world due to DM, $80 \%$ of these cases are preventable (10). The foot lesions are often chronic and resistant to treatment. These ulcers are prone to secondary infections; bacterial, fungal and viral (11). Poor controlled had the significantly higher fungal infection in diabetic foot ulcers and require careful attention and management (12). Persons with DM have a $15 \%$ to $25 \%$ chance of developing a diabetic foot ulcer (DFU) during their lifetime, and a $50 \%$ to $70 \%$ recurrence rate over the ensuing 5 years. More than half of persons with diabetes mellitus are unaware of their disease. $2.5 \%$ to $15 \%$ of annual global health care budgets are spent on diabetes mellitus. Diabetes mellitus is the seventh leading cause of death in the United States. Diabetes mellitus is the leading cause of kidney failure, non-traumatic lower extremity amputations, and new cases of blindness in adult Americans. Diabetic foot ulcers are often preventable, and treatment is frequently suboptimal. Chronic wounds, including DFUs, are a common yet challenging problem. Diabetic foot ulcers precede $85 \%$ of lower limb amputations. Diabetic foot ulcers are the most expensive and preventable complication of diabetes 
mellitus. The average lower limb amputation and rehabilitation cost $\$ 44,790$. Persons with DM are susceptible to peripheral neuropathy with sensory, autonomic, and motor components. Neuropathy is one of the main contributory factors in the pathogenesis of DFUs. Microand macro-vascular disease in persons with DM may impair healing of the ulcers and is critically important. Ischemia has been reported as a contributing factor in $90 \%$ of diabetic patients undergoing major amputation. Increased plantar pressure resulting from neuropathy is the major risk factor for diabetic foot ulcers. Neuropathy impairs the ability to perceive injury because of a loss of protective sensation. Autonomic neuropathy involves the sympathetic nervous system and presents as anhidrosis with dry skin and fissures that needs to be distinguished from other causes of dry plantar skin. These changes must be distinguished from a fungal infection because the fourth and fifth web spaces are common areas for fungal intertrigo while the plantar surface and the sides of the foot are common areas for a moccasin distribution. Diabetic foot ulcers can be divided into neuropathic, ischemic, and neuro-ischemic foot ulcers, with the latter 2 having a less favorable prognosis (13).

Fungal infections are usually neglected aspects of cutaneous lesions in DM patients. Foot infections are a frequent complication of patients with diabetes mellitus, accounting for up to $20 \%$ of diabetes-related hospital admissions. Bacteria are predominant in acute diabetic foot infections and that chronic infections may involve gram-negative bacteria and anaerobes. Recent South Indian studies have shown that gram-negative bacteria in diabetic foot ulcers are mostly monomicrobial (14).

Our findings indicated more than $80 \%$ of patients with diabetic foot ulcers had a history of trauma and burns and more than $50 \%$ of them had neuropathic ulcers. In Seattle, $46 \%$ of the amputations were related to ischemia, $59 \%$ to infection, $61 \%$ to neuropathy, $81 \%$ to faulty wound healing, $84 \%$ to ulceration, $55 \%$ to gangrene and $81 \%$ to initial minor trauma (15). Trauma and burns with an impaired leucocyte functions in diabetic patients could be the risk factors for the high prevalence of fungal infection at the recent studies (16-18).

Literature data on the frequency of fungal infection on the different type of cutaneous lesions is significantly different. Gupta et al showed that diabetic patients with onychomycosis were more at risk for diabetic ulcers and gangrene (12.2\%) than normal individuals with onychomycosis (19). C. albicans is the most common yeast isolated from the diabetic foot ulcer, skin and nail lesions and A. flavus was the most prominent causative agent of onychomycosis in DM patients in Isfahan (3). Over the past several decades, the incidence of nosocomial fungal infections (i.e., invasive fungal infections acquired in a healthcare-associated setting) has dramatically increased. The relative frequencies with which fungi causenosocomial infections are inversely related to the intensity of immunosuppression required to predispose to them. For example, relatively minimal Immunosuppression is required to predispose to invasive Candida infections, and Candida is by far the most common cause of nosocomial fungal infections. Aspergillus is the second most frequent cause of nosocomial fungal infections and aspergillosis tends to occur in patients with an intermediate to severe degree of immunocompromise. Finally, organisms such as the Mucorales, Fusarium, and other molds (e.g., Scedosporium) are relatively less common, and are seen virtually exclusively in the most severely immunocompromised hosts, and in hosts that are compromised for prolonged periods of time (20).Fungal infections of urinary tract infections are commonly caused by Candida spp and rarely present as "fungus ball.' Unusual organisms and gramnegative pathogens other than "E coll" may 
also occur in patients with diabetes mellitus. Patients with diabetes mellitus are at increased risk of superficial fungal infections like onychomycosis and tinea pedis. Mucormycosis is an uncommon, acutely fatal fungal infection that predominantly affects immunocompromised patients. It carries a high mortality ranging from $15 \%$ to $34 \%$. Rhinocerebral mucormycosis, the most common form of infection, predominantly affects patients with poorly controlled diabetes, especially with diabetic ketoacidosis. Patients with rhinocerebral mucormycosis with concomitant diagnosis of diabetes ranged from $60 \%$ to $81 \%$. Infections in diabetic patients result in extended hospital stays and additional financial burden(21).

Dorko et al, in a study on DM patients in Slovakia, found that, C. albicans was the most frequent yeast in patients with

\section{References}

1. Murphy-Chutorian B, Han G, Cohen SR. Dermatologic manifestations of diabetes mellitus: a review. Endocrinol Metab Clin North Am. 2013;42(4):86998. doi: 10.1016/j.ecl.2013.07.004.

2. Bagher L, Zahedi F. [Epidemiology of diabetes mellitus in Iran]. Iran J Diabetes Metab. 2001; 1 (1) :1-8. (Article in Persian)

3. Raiesi O, Siavash M, Mohammadi F, Chabavizadeh J, Mahaki B, Maherolnaghsh M, et al. Frequency of cutaneous fungal infections and azole resistance of the isolates in patients with diabetes mellitus. Adv Biomed Res. 2017; 6:71. doi: 10.4103/22779175.191003.

4. Singh N, Armstrong DG, Lipsky BA. Preventing foot ulcers in patients with diabetes. JAMA. 2005;293(2):217-28. doi:10.1001/jama.293.2.217.

5. Ricardo RG, Hector RM, Corando S. Mucormycosis: report of 11 cases. Arch Neurol. 1985;42(6):578-581. doi:10.1001/archneur.1985.040600600 80013. onychomycosis followed by $C$. parapsilosis (22).

\section{Conclusion}

The findings of various studies showed a high prevalence of fungal infection in diabetic and diabetic foot ulcer patients and C. albicans was the prominent fungus isolated from these patients. Wise consideration of the possibility of fungal infections, early recognition, and appropriate treatment ensure rapid healing and eliminate amputation risk, minimize mortality and costs.

\section{Acknowledgments}

Sincere gratitude of all professors and students of mycology at the School of Public Health in Tehran University of Medical Sciences.

6. Robert IL, Dexter HH, Paul SS, John EE, Gary PS, Drew JW. Mucormycosis. Ann Intern Med. 1980;93(1):93-108. doi: 10.7326/0003-4819-93-1-93.

7. Mehra BK. A Clinicomicrobiological study on incidence of Mycotic infections in diabetic foot ulcers. Inter $\mathbf{J}$ Sci Stud. 2017;4(12): 50-4.

8. Chayakulkeeree M, Ghannoum MA, Perfect JR. Zygomycosis: the reemerging fungal infection. Eur J Clin Microbiol Infect Dis. 2006;25(4):21529.

9. Romano G, Moretti G, Di Benedetto A, Giofrè C, Di Cesare E, Russo G, et al. "Skin lesions in diabetes mellitus: prevalence and clinical correlations. Diabetes Res Clin Pract. 1998;39(2):101-6.

10. Bakker K, van Houtum WH, Riley PC. 2005: The International Diabetes Federation focuses on the diabetic foot. Curr Diab Rep. 2005;5(6):436-40.

11. Aye M, Masson EA. Dermatological care of the diabetic foot. Am J Clin Dermatol. 2002;3(7):463-74. 
12. Chellan G, Shivaprakash S, Karimassery Ramaiyar S, Varma AK, Varma N, Thekkeparambil Sukumaran $\mathrm{M}$, et al. Spectrum and prevalence of fungi infecting deep tissues of lowerlimb wounds in patients with type 2 diabetes. J Clin Microbiol. 2010;48(6):2097-102. doi: 10.1128/JCM.02035-09.

13. Alavi A, Sibbald RG, Mayer D, Goodman L, Botros M, Armstrong DG, et al. "Diabetic foot ulcers: Part I. Pathophysiology and prevention. J Am Acad Dermatol. 2014;70(1):1. e1-18; quiz 19-20. doi: 10.1016/j.jaad.2013.06.055.

14. Shankar EM, Mohan V, Premalatha G, Srinivasan RS, Usha AR. Bacterial etiology of diabetic foot infections in South India. Eur J Intern Med. 2005;16(8):567-70.

15. Pecoraro RE, Reiber GE, Burgess EM. Pathways to diabetic limb amputation: basis for prevention. Diabetes Care. 1990;13(5):513-21.

16. Borzotta AP, Beardsley K. Candida infections in critically ill trauma patients: a retrospective case-control study. Arch Surg. 1999 Jun;134(6):657-
64; discussion

664-5.

doi:10.1001/archsurg.134.6.657.

17. Church D, Elsayed S, Reid O, Winston B, Lindsay R. Burn wound infections. Clin Microbiol Rev. 2006;19(2):40334 doi: 10.1128/CMR.19.2.403434.2006.

18. Delamaire M, Maugendre D, Moreno M, Le Goff MC, Allannic H, Genetet B. Impaired leucocyte functions in diabetic patients. Diabet Med. 1997;14(1):29-34.

19. Gupta AK, Konnikov N, MacDonald P, Rich P, Rodger NW, Edmonds MW, et al. Prevalence and epidemiology of toenail onychomycosis in diabetic subjects: a multicentre survey. $\mathrm{Br} \mathrm{J}$ Dermatol. 1998;139(4):665-71.

20. Perlroth J, Choi B, Spellberg B. Nosocomial fungal infections: epidemiology, diagnosis, and treatment. Med Mycol. 2007 Jun;45(4):321-46.

21. Gupta S, Koirala J, Khardori R, Khardori N. Infections in diabetes mellitus and hyperglycemia. Infect Dis Clin North Am. 2007;21(3):617-38.

22. Dorko E, Baranová Z, Jenca A, Kizek P, Pilipcinec E, Tkáciková L. Diabetes mellitus and candidiases. Folia Microbiol (Praha). 2005;50(3):255-61. 